BMC

Evolutionary Biology

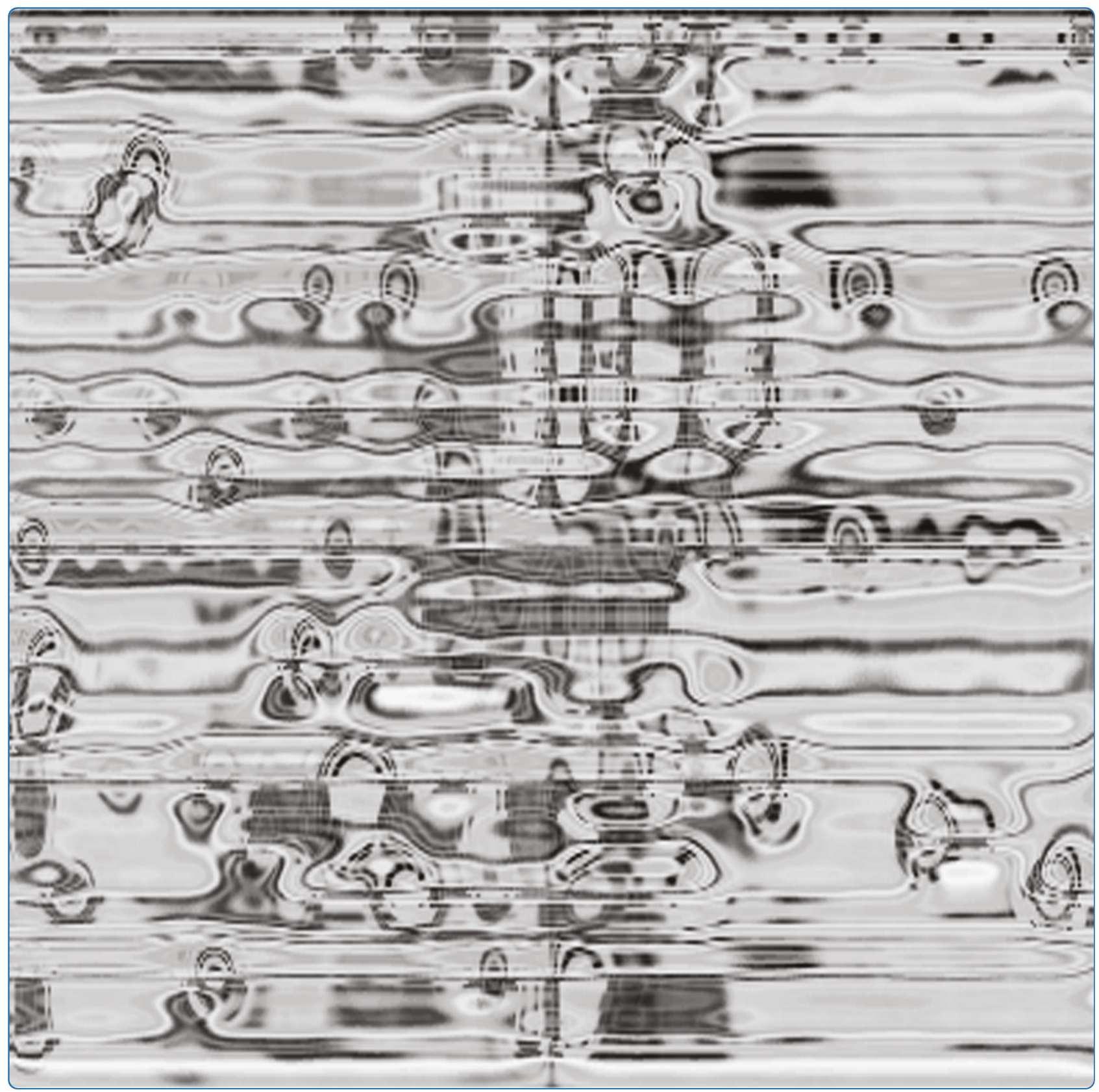

\title{
Evolutionary relationships and diversification of barhl genes within retinal cell lineages
}

Schuhmacher et al. 


\title{
Evolutionary relationships and diversification of barhl genes within retinal cell lineages
}

Laura-Nadine Schuhmacher $^{1 \dagger}$, Shahad Albadri ${ }^{1 \dagger}$, Mirana Ramialison ${ }^{1,2}$ and Lucia Poggi ${ }^{*}$

\begin{abstract}
Background: Basic helix-loop-helix and homeodomain transcription factors have been shown to specify all different neuronal cell subtypes composing the vertebrate retina. The appearance of gene paralogs of such retinaspecific transcription factors in lower vertebrates, with differently evolved function and/or conserved non-coding elements, might provide an important source for the generation of neuronal diversity within the vertebrate retinal architecture. In line with this hypothesis, we investigated the evolution of the homeobox Barhl family of transcription factors, barh/1 and barh/2, in the teleost and tetrapod lineages. In tetrapod barh/2, but not barh/1, is expressed in the retina and is important for amacrine cell specification. Zebrafish has three barhl paralogs: barhl1.1, barhl1.2 and barh/2, but their precise spatio-temporal retinal expression, as well as their function is yet unknown.

Results: Here we performed a meticulous expression pattern comparison of all known barhl fish paralogs and described a novel barh/ paralog in medaka. Our detailed analysis of zebrafish barh/ gene expression in wild type and mutant retinas revealed that only barh/1.2 and barh/2 are present in the retina. We also showed that these two paralogs are expressed in distinct neuronal lineages and are differently regulated by Atoh7, a key retinal-specific transcription factor. Finally, we found that the two retained medaka fish barh/ paralogs, barh/1 and barh/2, are both expressed in the retina, in a pattern reminiscent of zebrafish barh 1.2 and barh/2 respectively. By performing phylogenetic and synteny analysis, we provide evidence that barh/ retinal expression domain is an ancestral feature, probably lost in tetrapods due to functional redundancy.

Conclusions: Functional differences among retained paralogs of key retina-specific transcription factors between teleosts and tetrapods might provide important clues for understanding their potential impact on the generation of retinal neuronal diversity. Intriguingly, within teleosts, retention of zebrafish barh/1.2 and its medaka ortholog barh/1 appears to correlate with the acquisition of distinct signalling mechanisms by the two genes within distinct retinal cell lineages. Our findings provide a starting point for the study of barhl gene evolution in relation to the generation of cell diversity in the vertebrate retina.
\end{abstract}

\section{Background}

The vertebrate retina is organized into a complex network of cell layers, namely the ganglion cell layer (GCL) which contains retinal ganglion cells (RGCs) and displaced amacrine cells (ACs), the inner nuclear layer (INL) which consists of ACs, horizontal, bipolar and Müller glia cells, and the outer nuclear layer (ONL) which is made up of cone and rod photoreceptors. This strikingly complex architectural plan of the retina is

\footnotetext{
* Correspondence: lucia.poggi@cos.uni-heidelberg.de

+ Contributed equally

'Centre for Organismal Studies, University of Heidelberg, Heidelberg 69120, Germany

Full list of author information is available at the end of the article
}

extremely well conserved across vertebrate species, probably in direct correlation with the conservation of the key regulatory factors that govern retinal development. Several members of the basic helix-loop-helix (bHLH) and homeodomain family of transcription factors are known to play a role in the determination of retinal progenitor competence and cell fate, a function that is highly conserved from fish to mammals [1]. Much less is known on the contribution of different functional paralogs of retina-specific transcription factors, which arose subsequently to rounds of whole genome duplication (WGD) during vertebrate evolution [2]. Indeed, it has been proposed that after WGD, duplicated genes can either accumulate loss-of-function
C Biomed Central

(c) 2011 Schuhmacher et al; licensee BioMed Central Ltd. This is an Open Access article distributed under the terms of the Creative Commons Attribution License (http://creativecommons.org/licenses/by/2.0), which permits unrestricted use, distribution, and reproduction in any medium, provided the original work is properly cited. 
mutations and are functionally lost (non-functionalization $[3,4]$ ) or acquire a new function (neo-functionalization), or split the ancestral function between the paralogs (sub-functionalization) [2]), therefore adding complexity to the developmental gene network that shapes organ formation. The genes of the barhl family encoding the homeobox transcription factors Barhl1 and Barhl2, have been shown to be expressed in more or less overlapping domains of the central nervous system and have partially redundant functions in neural subtype cell identity, migration and survival [5,6]; however, barhl2 members appear to be uniquely expressed in the retina $[7,8]$. In particular, Barhl2 is a pan-vertebrate regulator of the specification and survival of ACs and RGCs [9-11]. Forced expression of Barhl2 in the mouse retina promotes the differentiation of glycinergic amacrine cells at the expense of bipolar and Müller cells [10]. Additionally, analysis of Barhl2-null retinas suggests that Barhl2 plays a critical role in both AC subtype determination and in RGC survival [9]. The Xenopus Barhl2 ortholog (previously named Xbh1) has been shown to be expressed in RGCs and in presumptive AC precursors, and to promote RGC differentiation downstream of the bHLH transcription factor Atoh7 [11]. While Xenopus, mouse, rat and human have one copy of barhl1 and barhl2 each, zebrafish has three barhl paralogs possibly due to a further genome duplication event that teleosts underwent during evolution after the split from the tetrapod lineage $[12,13]$. On the basis of protein sequence alignment and phylogenetic analysis, it has been suggested that two of these orthologs belong to the barhl1 paralog group (nominated barhl1.1 and barhl1.2) while the third belongs to the barhl2 group $[6,12]$. In contrast to mouse and Xenopus, all three barhl seem to be expressed both in the brain and in the retina [12]. In medaka fish (Oryzias latipes), only one barhl has been described so far (olbarhl); based on phylogenetic analysis olbarhl has been clustered to the barhl1 group of paralogs $[6,12,14]$. To get more insights into the evolution of barhl paralogs with respect to retinal differentiation we took advantage of the zebrafish and medaka model systems to perform a comprehensive comparative analysis of barhl gene expression as compared to the one in tetrapod. By detailed in situ hybridization analysis we confirmed that barhl1.2 and barhl2 are expressed in the zebrafish retina but not barhl1.1. A meticulous inspection of barhl1.2 and barhl2 transcript distribution indicates that while barhl2 appears to recapitulate the expression of its mammalian and Xenopus counterpart, the spatio-temporal expression pattern of barhl1.2 is non redundant to that of barhl2, suggesting that barhl1.2 might have evolved non redundantly with respect to barhl2 in the retina. Interestingly, we have detected that barhl1.2 shows a very early onset of expression which is highly overlapping with the expression of the atoh7 gene demarcating the onset of RGC genesis. Furthermore, we also describe a new barhl paralog in medaka which, based on protein alignment, could be assigned to the Barhl2 group. By comparing the expression of medaka barhl1 and barhl2, we found that they are both expressed in the retina, in a pattern reminiscent of zebrafish barhl1.2 and barhl2 respectively. These results combined with phylogenetic and synteny analysis suggest that barhl retinal expression domain is an ancestral feature that has been specifically lost in tetrapod probably due to functional redundancy following the duplication-supplementation paradigm $[3,4]$.

\section{Results}

Zebrafish barh/1.2 and barh/2, but not barh/1.1, are expressed in distinct spatio-temporal domains of the developing retina

In light of the distinct expression of barhl1 and barhl2 in the tetrapod retina, we aimed at clarifying the retinal expression domains spanned by each of the three paralogs to get further insight into a possible non-redundant function of barhl paralogs in the zebrafish retina. We performed in situ hybridization on embryos at different developmental stages, starting from the beginning of retinal differentiation around 30 hours-post-fertilization (hpf), until $70 \mathrm{hpf}$. Transcripts of barhl2 are detected in the retina as early as $35 \mathrm{hpf}$ (Figure 1B). At this stage, most RGCs and the first ACs are born [15]. Barhl2 transcripts are initially localized in a few cells in the central retina and subsequently expressed in the whole retina (Figure 1A-C), in a pattern matching the wave of AC genesis. At $40 \mathrm{hpf}$, expression appears mostly restricted to the inner part of the INL (Figure 1J) and later on it extends to the GCL (Figure 1C, K). By $70 \mathrm{hpf}$ expression is maintained in both the INL and the RGC layer (data not shown). Interestingly, transcripts of barhl1.2 were detected in the retina already at 28-30 hpf (Figure 1D). This stage corresponds to the peak of RGC genesis. Similarly to barhl2, its expression is initially restricted to the central retina and subsequently spreads temporally and dorsally (Figure 1D, E). However, this expression appears largely restricted to the RGC layer (Figure $1 \mathrm{E}, \mathrm{L}$ and $1 \mathrm{M})$ and it later becomes restricted to the ciliary marginal zone (CMZ) (50 hpf, Figure 1F). In contrast, no evident expression of barhl1.1 was found in the retina at all stages analyzed, although transcripts could be clearly detected in the brain (Figure 1G-I). This observation confirms that zebrafish barhl1.1 is the ortholog of other vertebrate barhl1; which were never found expressed in the retina of tetrapod $[7,8]$. 


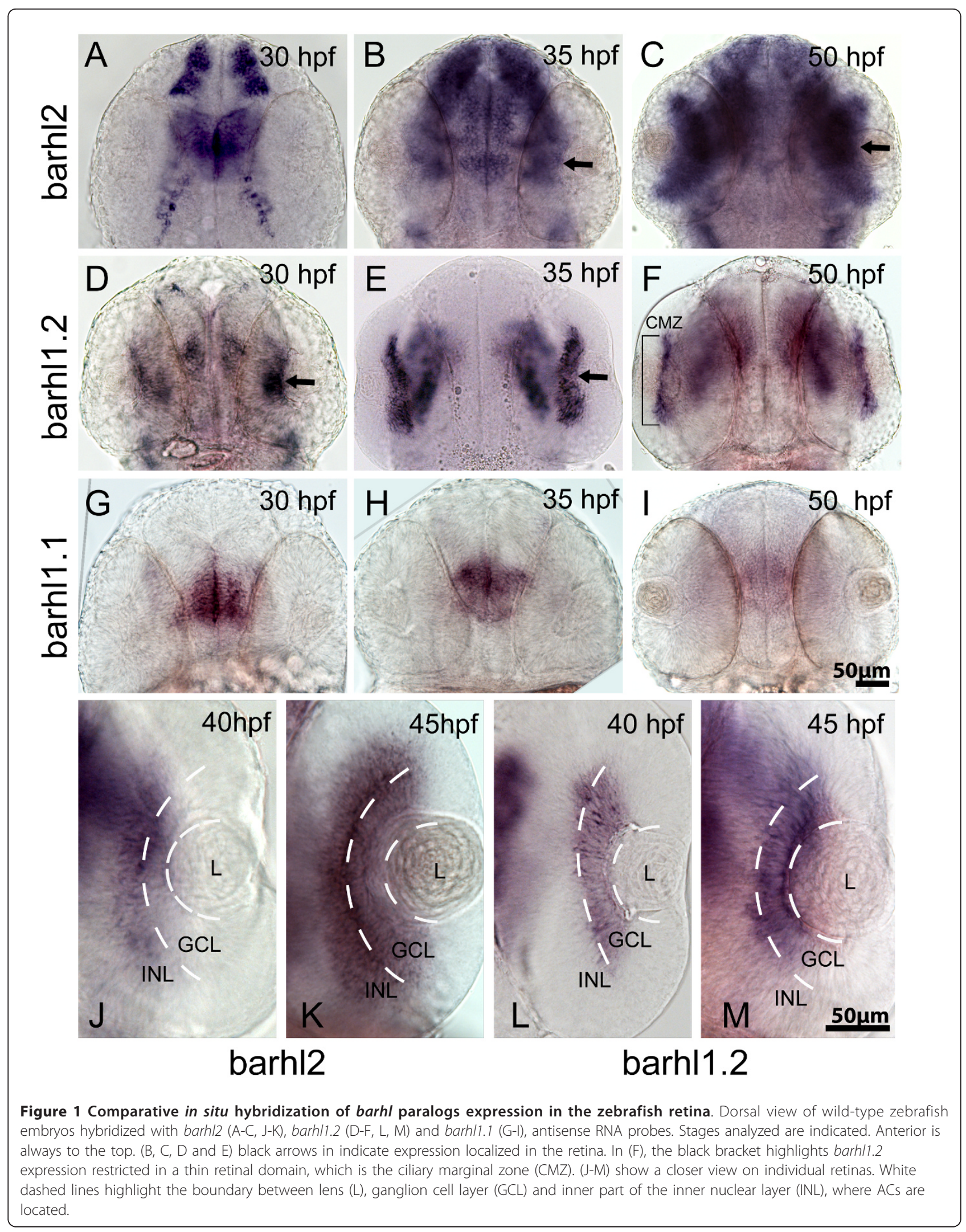




\section{Expression of barhl1.2 and barh/2 in the retina suggests distinct regulation by atoh7}

Given the observed non-redundant expression of barhl2 and barhl1.2 transcripts in the retina, we then investigated in greater detail the relative spatio-temporal distribution of these two transcripts by double fluorescent in situ hybridization (FISH). At 35 hpf, a few barhl2-FITC (in green) positive cells could be detected in the central retina, located within a broader domain of barhl1.2-Cy3 (in red) positive cells (Figure 2A, D and 2G). Within this domain, expression of barhl1.2 and barhl2 appears mostly non-overlapping (white arrows in Figure 2A, D and 2G). By $40 \mathrm{hpf}$, transcripts of barhl1.2 are mostly restricted to the GCL while the ones of barhl2 are mostly found in the inner part of the INL where ACs are present (Figure $2 \mathrm{~B}, \mathrm{E}$ and $2 \mathrm{H}$ ). Interestingly, overlapping and distinct patterns of expression of barhl1.2 and barhl 2 can be observed also in the brain (see example in the diencephalon in Figure 2C, F and 2I).

To map out the temporal and spatial relationship between barhl1.2 and barhl2 expression and RGC differentiation, we compared their expression to the one of atoh7 [16]. The proneural bHLH (basic helix-loop-helix) transcription factor Atoh7 (previously named Ath5), homolog of Drosophila Atonal, has been shown to be essential for RGC differentiation in mouse, zebrafish and human [17-20] and is transiently expressed in retinal precursors fated to become mainly RGCs, but also horizontal, photoreceptor and specific subpopulations of ACs $[15,21,22]$. FISH performed with barhl1.2-Cy5 (in red) and atoh7-FITC (in green) shows that at $30 \mathrm{hpf}$ transcripts of both genes co-localize in the central retina (Figure 3A-C). At $40 \mathrm{hpf}$, atoh7 transcripts start to be downregulated in the mature RGCs of the central retina (Figure 3E, F), while barhl1.2 expression is maintained in this area (Figure 3D-F). At this stage, we begin to observe a co-localization of barhl1.2 with atoh7 within the CMZ (Figure 3F) clearly visible at 50hpf, (Figure 3G-I). Thus, barhl1.2 expression overlaps and follows the expression of atoh7 with a slight delay, suggesting that atoh7 might directly or indirectly influence expression of barhl1.2 in progenitors adopting the RGC fate. We then analyzed the expression of barhl2 with respect to the one of atoh7. Interestingly, in this case barhl2 expression is mostly complementary to the one of atoh7 (Figure 4). At $35 \mathrm{hpf}$, barhl2 starts to be expressed in a central retinal domain where atoh 7 transcripts are already downregulated (Figure 4A-E). Later on, barhl2 expression expands towards the peripheral retina in a fan-like wave while atoh 7 gets restricted to the CMZ (see $45 \mathrm{hpf}$ and $50 \mathrm{hpf}$ in Figure $4 \mathrm{~F}-\mathrm{J}$ and $4 \mathrm{~K}-\mathrm{O}$ respectively). At all stages analyzed, barhl2 and atoh7 expressions appear mostly mutually exclusive (examples are highlighted with asterisks in Figure 4I-J, N-O), although few cells co-expressing both genes are always visible at the expression boundaries (as indicated by arrows in Figure 4D-E, and 4N-O). Therefore, in contrast with barhl1.2, the complementary expression of barhl 2 and atoh 7 suggests a reciprocal negative regulation between these two genes. To further investigate this aspect in vivo, we took the advantage of the atoh7-/- mutant embryos (lakritz). In the atoh7-/- retina RGCs fail to exit the cell cycle and to differentiate as a result of a loss-of-function mutation within the atoh7 gene [19]. We tested how atoh7 loss-of-function and therefore lack of RGC differentiation affect the expression of barhl1.2 and barhl2 (Figure 5). In situ hybridization on embryos at $40 \mathrm{hpf}$ shows that barhl1.2 transcripts are completely missing in the GCL of the atoh7-/- retina (Figure 5E-F), while barhl2 expression is retained in this mutant retina (Figure $5 \mathrm{~A}-\mathrm{B}$ ). The expression of both barhl paralogs in other brain areas, such as the tectum and the rhombic lips, is not affected by Atoh7 loss (Figure 5C-D and 5 G-H). Thus, barhl1.2 appears to be sustained by atoh7 while barhl2 onset of expression appears independent on atoh7. It remains to be demonstrated whether a negative feedback interaction exists between barhl2 and atoh7.

\section{Identification of a novel barhl paralog in medaka}

As it is commonly accepted that all teleosts underwent one further round of WGD, we wanted to test whether other teleosts have also retained more than two barhl and how their expression in the retina evolved. The medaka fish is a well-established model system and is therefore very suitable for a comparison with zebrafish [23]. So far, one barhl has been described in the medaka fish (olbarhl, [14]); based on phylogenetic analysis olbarhl has been clustered within the Barhl1 group of paralogs (olbarhl [6]). Interestingly, olbarhl1 is also expressed in the retina and in particular in the developing GCL [14]. In order to identify other putative barhl paralogs, we performed a BLAST search against the medaka genome (EnsEMBL Release 58, see methods) using zebrafish Barhl proteins as queries. We could identify a second member of the medaka barhl family on chromosome 4. By performing a multiple sequence alignment of Barhl proteins, the newly identified medaka Barhl was assigned to the Barhl2 group (Figure 6). The newly identified Barhl medaka protein has an asparagine residue instead of an alanine at position 15, an aspartic acidic residue instead of a glutamic acid at position 34 as well as an alanine instead of serine at position 38 (highlighted by asterisks in Figure 6C). The homeobox sequences of vertebrate Barhl differ at these positions and clearly divide the genes in the two mentioned groups. The FIL domains represent another set of highly conserved motifs $[7,8,12,14,24]$. Only one of the FIL domains, FIL2, can be found in both medaka Barhl1 and Barhl2 (Figure 


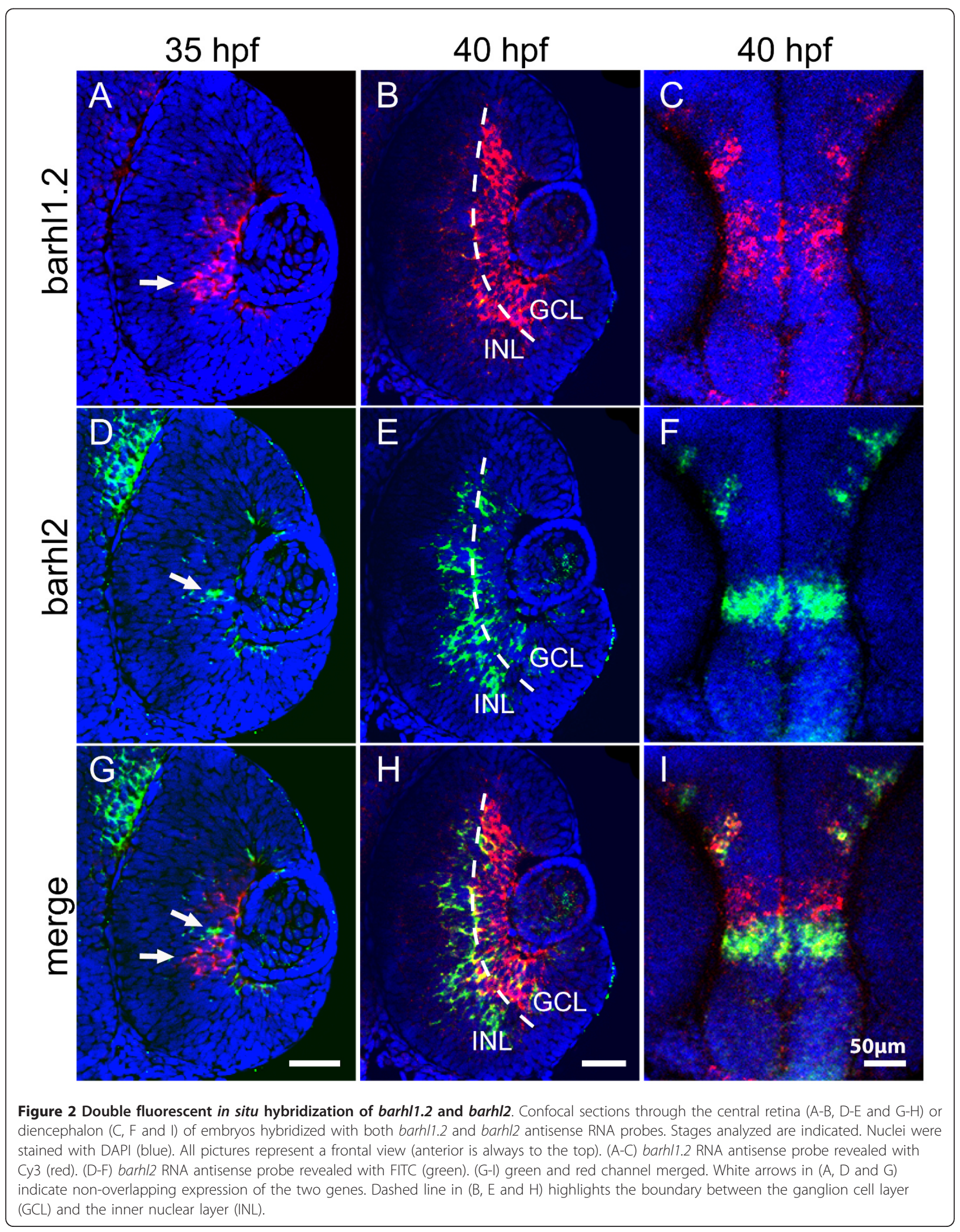




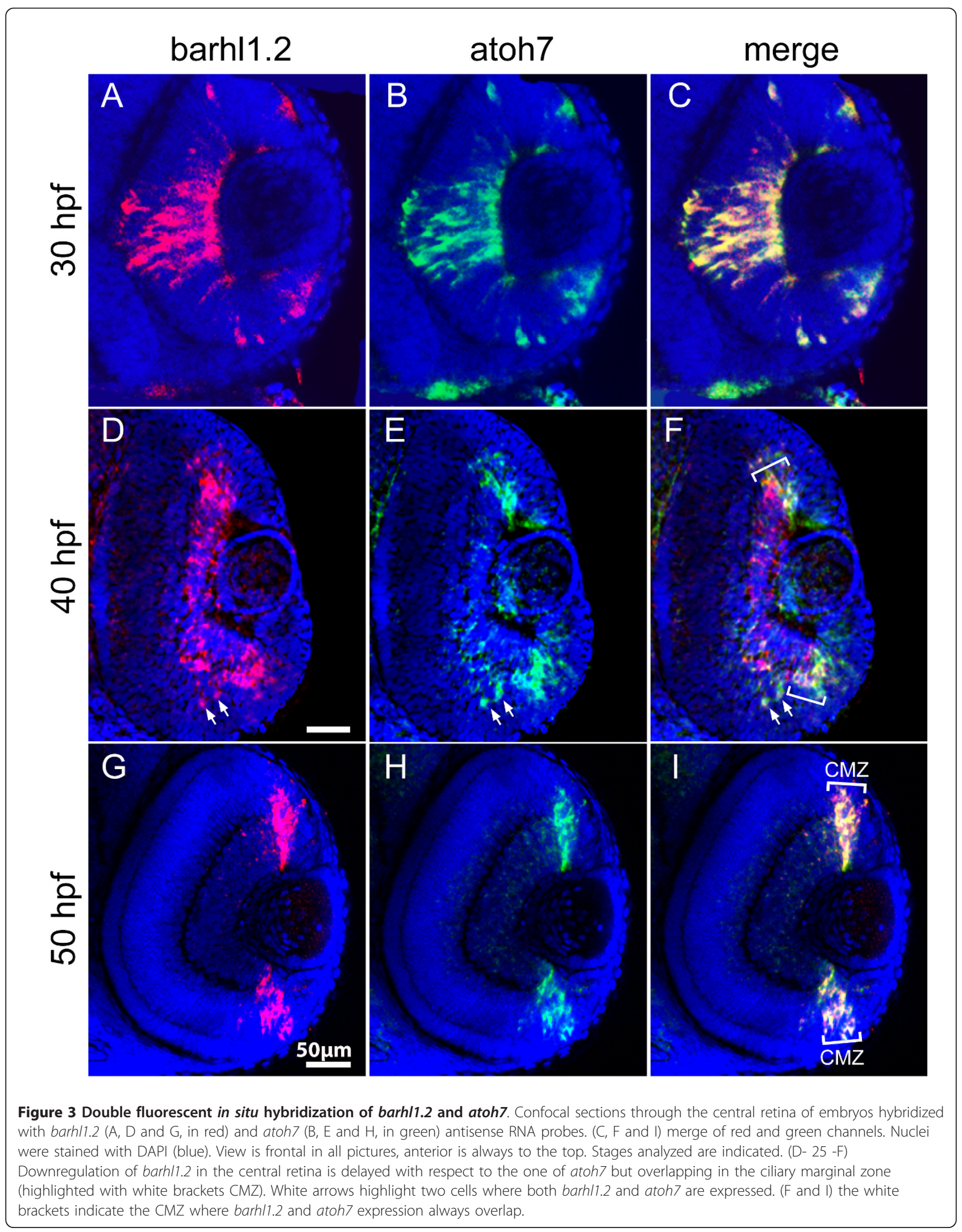


$6 \mathrm{~A}, \mathrm{~B})$. To further characterize the evolutionary origin of the new medaka ortholog, a phylogenetic tree was constructed using Drosophila BarH1 and BarH2 protostome outgroup (Figure 7). Additionally, we rooted the tree with Barhl protein sequences of the ancestral deuterostomes Ciona savignyi and Branchiostoma floridae, which we identified by BLAST search. Ciona savignyi Barhl and Branchiostoma floridae Barhl also cluster with the outgroup, as those two deuterostomes have not undergone the vertebrate-specific WDG and have only one ancestral Barhl protein. The Barhl proteins are clearly clustered in two groups, with the newly identified medaka sequence belonging to the Barhl 2 group. Thus, medaka has one Barhl1 and one Barhl2 that relate to the homologs in other teleosts, as we would expect from the relationship among these teleost species. Our tree clearly illustrates the relationship between zebrafish Barhl1 paralogs and other teleosts Barhl1 proteins and suggests that zebrafish Barhl1.2 has split basally from the teleost Barhl1 group.

We then sought to compare the expression patterns of both medaka barhl1 and medaka barhl2 in the retina by in situ hybridization, using antisense probes against transcripts amplified from mixed stages-brain and eye cDNA (see Methods). Medaka barhl1 starts to be expressed in the central retina at stage $25[14,25]$. This stage more or less corresponds to $28-30 \mathrm{hpf}$ in zebrafish, when atoh7 expression and RGC differentiation begin $[23,26]$. Later on, expression extends to the whole RGC layer [14]. We found that at stage 30, the medaka barhl1 is still expressed in the GCL but is already being

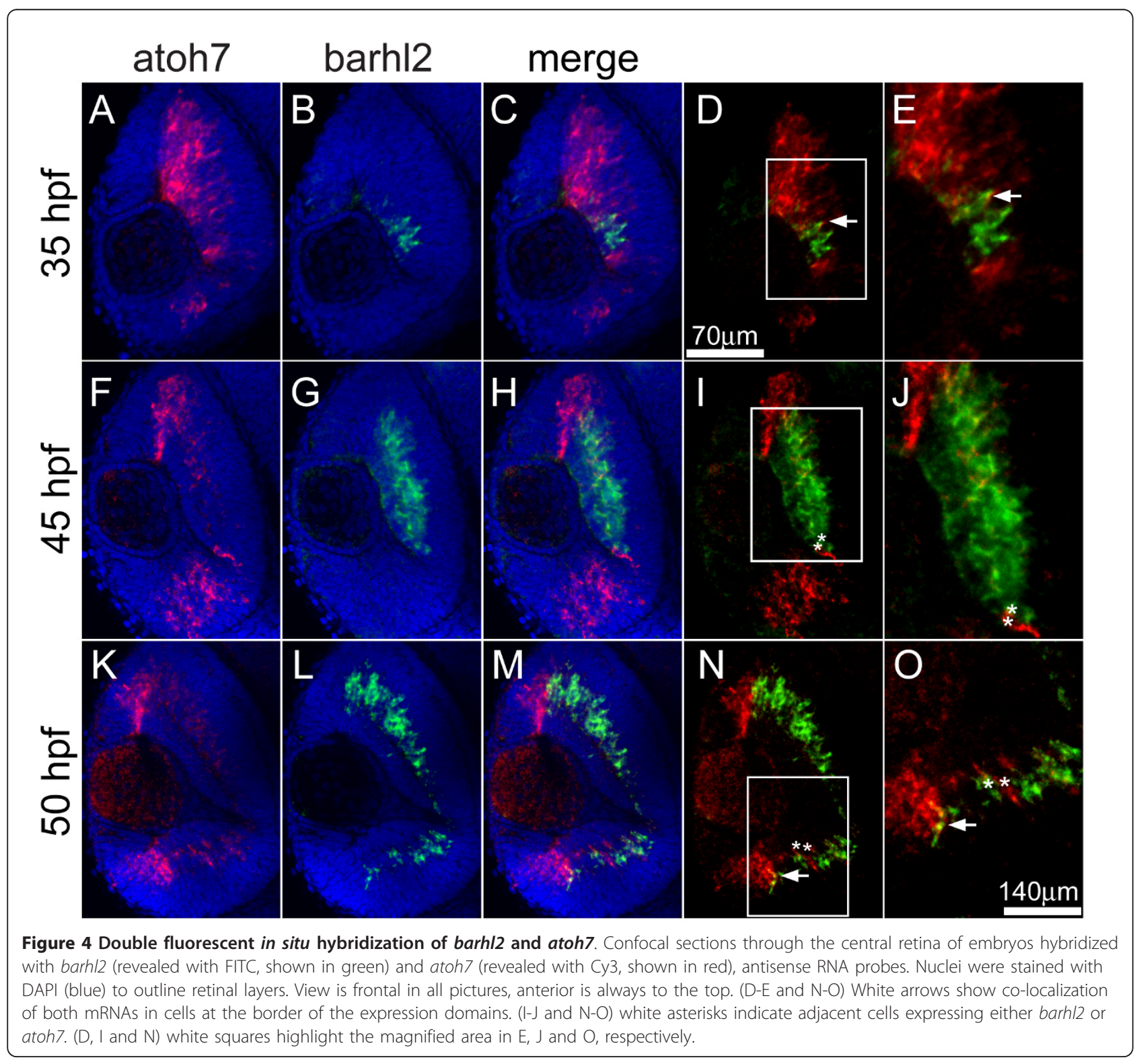




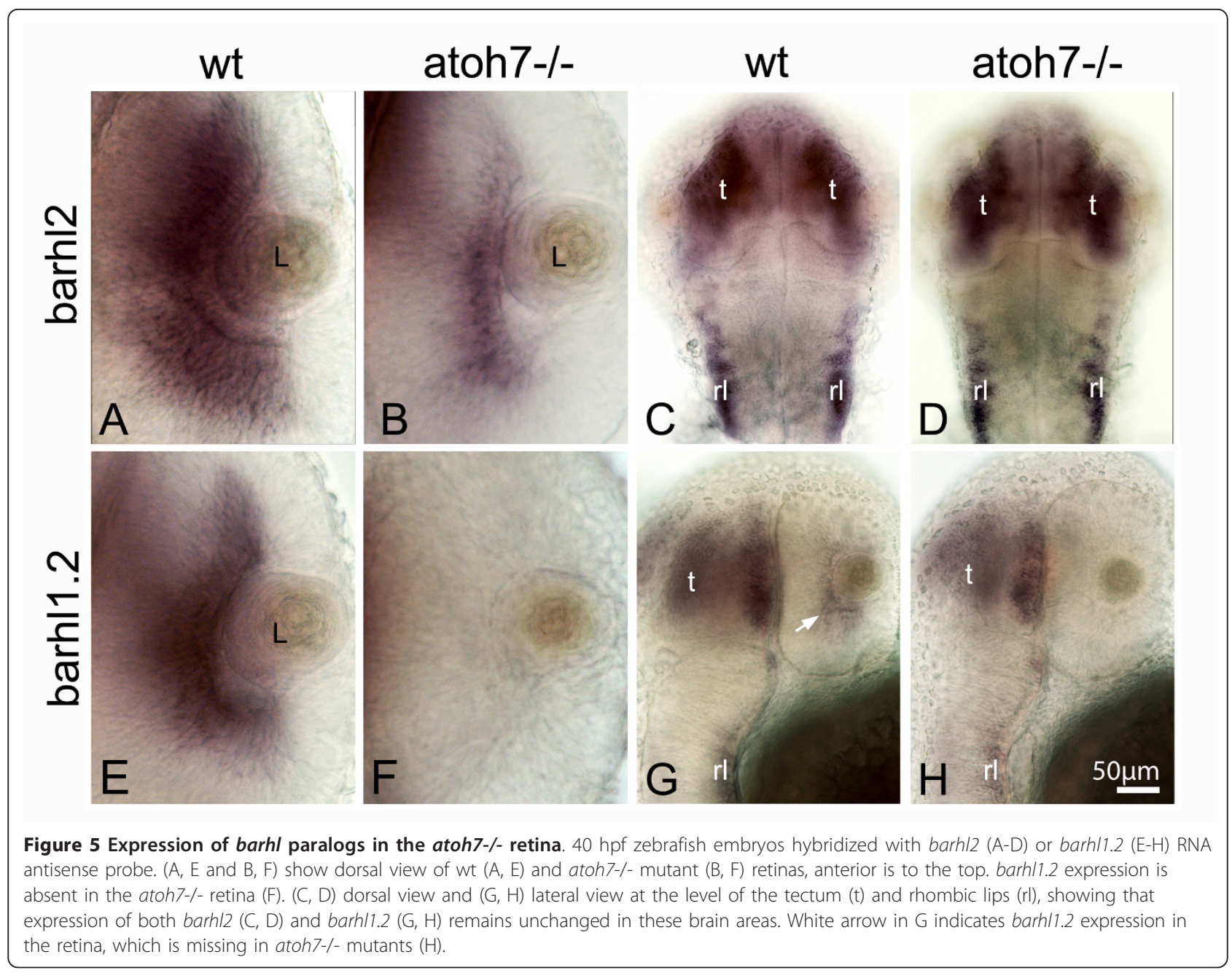

downregulated in the central retina (Figure 8A). Interestingly, this is the stage at which atoh7 expression also starts to be downregulated in the central retina in medaka [26]. On the other hand, the medaka barhl2 is still strongly expressed in the central retina (Figure 8C). By stage 35, medaka barhl1 transcripts can be detected only in the CMZ (Figure 8B) while medaka barhl2 is still strongly expressed in both the GCL and the inner part of the INL (Figure 8D). Thus, the expression domain of the medaka barhl1 appears very similar to that of the zebrafish barhl1.2 as both transcripts can be found very early in the GCL and are very soon after restricted to the CMZ. Conversely, the medaka expression of barhl2 resembles the one observed for zebrafish barhl2, as both can be found strongly expressed in the INL and GCL until late stages of development.

\section{Conserved gene synteny between barhl genes}

Has the retinal expression domain been lost by one of the barhl duplicates, or has it been acquired after divergence of the two duplicated genes? Since the teleost branch underwent a WGD that did not affect other vertebrates, we expect to find two loci for each barhl gene in fish, which would explain the presence of two barhl1 paralogs in zebrafish, but only one paralog would have been retained in medaka. To further investigate this hypothesis, we compared the loci of barhl genes and looked for genes that are conserved in synteny. It has been proposed that genes that play important roles in development are surrounded by highly conserved elements and also show highly conserved gene synteny [27]. Kikuta et al. elaborated on the highly conserved synteny between the human and zebrafish barhl1 loci that might extend to the regulatory level [27]. For a more in-depth analysis we used additional teleost species to take into account syntenic conservation within these rapidly evolving fish species [28]. We searched for genes that are in synteny between zebrafish, stickleback, medaka, Tetraodon and human, and found that for both barhl2 and barhl1, the conservation of the locus is very 
high, both for non-coding sequence as well as syntenic genes. Genomic locations of all genes used in the analysis can be found in the Additional file 1: Table1. Figure 9A shows a model of the synteny relations between medaka, zebrafish and human. Human barhl1 is located on chromosome 9. Orthologs of the genes surrounding barhl1 in human can be found on zebrafish chromosomes 5 (where barhl1.2 is present) and 21 (where barhl1.1 is present) and medaka chromosomes 9 (where no barhl can be found) and 12 (where barhl1 is present). This scenario is most likely the result of WGD in teleosts and highlights that the second medaka barhl1 paralog, expected to be conserved in synteny on chromosome 9 has been lost during evolution. For barhl2 we can observe a similar pattern: The genes surrounding barhl2 on human chromosome 1 can be found distributed between two chromosomes each in zebrafish and medaka. Notably, in both teleost species only one duplicate of barhl2 has been retained. The fact that we found three or more genes that are conserved in their position as neighbours of barhl2 in loci that did not contain barhl2 (zebrafish chromosome 2, medaka chromosome 17) suggests that these chromosomal regions represent duplicated regions that did not retain a second barhl2 paralog. The loss of one barhl2 copy has been consistently observed in other teleosts species such as Tetraodon and fugu whereas the barhl1 locus seems to have undergone different genomic rearrangements. Indeed, in 


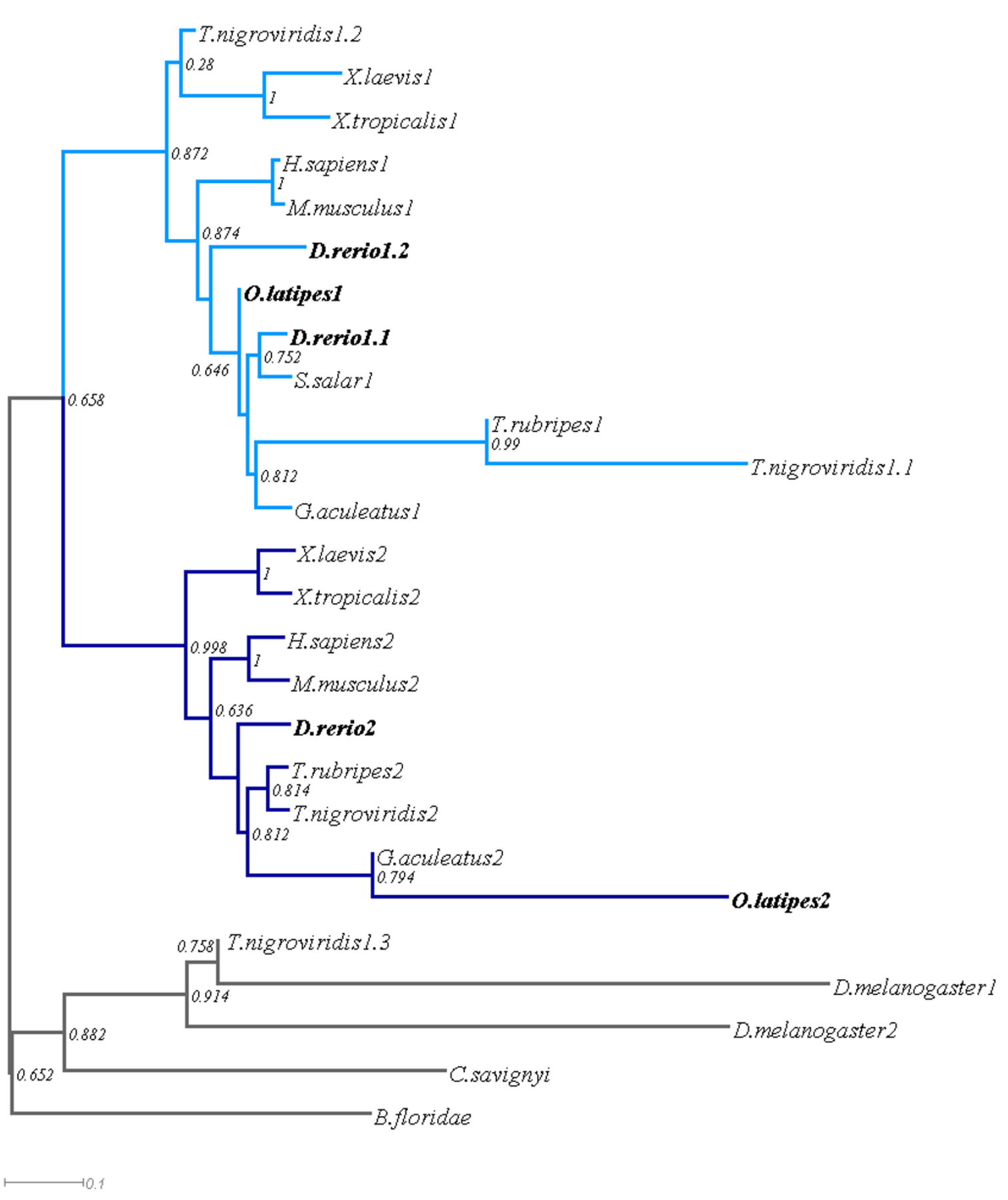

Figure 7 Phylogenetic tree of Barhl homologs based on protein sequence multi-alignment. A phylogenetic tree was inquired from the multiple sequence alignment using the neighbour-joining algorithm BioNJ. Barhl1 cluster is coloured in light blue, Barhl2 cluster in dark blue. Names related to zebrafish and medaka sequences are highlighted in bold. The numbers indicate the paralogs numbers for each species. Node values represent bootstrap support (bootstrap $=1000)$.

Tetraodon we found that syntenic genes around barhl1 are distributed to three distinct loci, whereas in stickleback, they can be found in a single linkage group (see Figure 9B for an overview on the distribution of the syntenic genes surrounding the barhl locus in all species considered in this analysis). Altogether, the evolution of the barhl1 locus in teleosts, coupled to WGD and species-dependent regional duplication, seems to be to be 


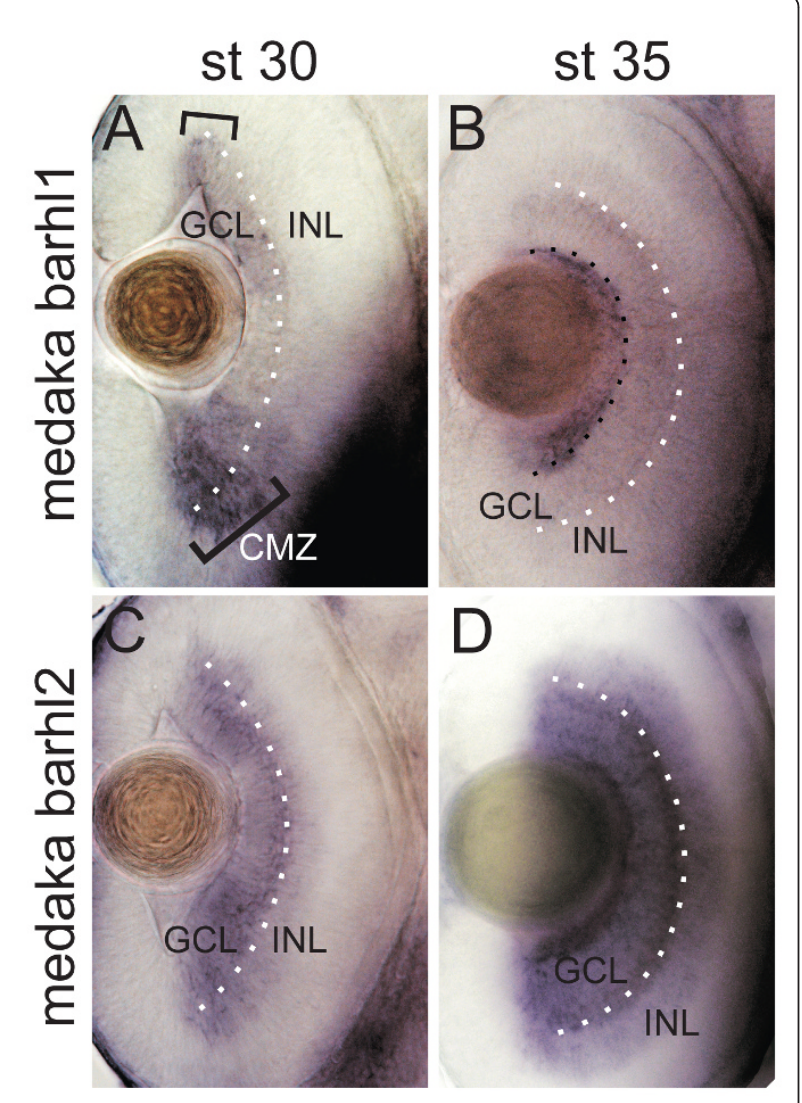

Figure 8 Expression patterns of the medaka barh/1 and barh/2 in the retina. Dorsal views through the retina of medaka embryos hybridized with either medaka barh/1 (A, B) or medaka barh/2 (C, D) RNA antisense probe. Anterior is to the top. (A) Expression of medaka barh/1 restricted to the ciliary marginal zone (CMZ) is highlighted with black brackets in A and with a black dotted line in B. The white dotted line indicates the ganglion cell layer (GCL)/inner nuclear layer (INL) boundary.

under less evolutionary constraint than in the case of barhl2, where a stereotypic pattern of gene distribution can be observed.

\section{Discussion}

According to this study, we propose that vertebrates have two homologs of barhl (barhl1 and barhl2) due to WGD events that occurred before the emergence of vertebrates. This is in contrast with Drosophila, where two BarH genes probably arose from tandem duplication in the same locus. It is a topic of speculation whether there have been one or two rounds of WGD in early vertebrate evolution before the split of the teleosts from the other jawed vertebrates, and currently the most widely accepted view is that there were two $[29,30]$. Our findings are consistent with the latter view as the third paralog found in zebrafish most likely originated from the additional round of whole genome duplication that marked the rise of teleosts and did not affect other vertebrates [31]. This hypothesis is in agreement with the fact that in basal deuterostomes such as sea squirt and amphioxus, only one barhl locus could be found by BLAST search. From this speculation one would assume that all teleost fish once had at least three, maybe even four paralogs of barhl, some of which were lost. We found that in zebrafish both barhl2 and barhl1.2 are expressed in the developing retina. Barhl1.1, like other vertebrate barhl1 homologs, is not expressed in the retina whereas the medaka barhl1 and barhl2 are both expressed in the retina in a similar fashion as barhl1.2 and barhl2 in zebrafish, thus suggesting that medaka barhl1 has a similar dynamic of transcriptional regulation as zebrafish barhl1.2. Given that the barhl expression in the retina is an ancestral feature that was already present in Drosophila, the most parsimonious explanation for barhl1 gene evolution is that its retinal expression was maintained within the teleost lineage but was lost in Xenopus and mammals. Thereafter within teleosts, the zebrafish barhl1.1 also lost its retinal expression probably due to a redundant function with barhl1.2 and relaxed evolutionary pressure in its locus. This can be further illustrated in the context of the Tetraodon barhl1 paralogs. These sequences do only resemble partial or split Barhl proteins and therefore the positions in the tree are excluded from the teleost barhl1 cluster. We have called the split sequences Tetraodon Barhl1.2 and Barhl1.3 and the protein that clusters within the teleost group Barhl1.1. In contrast, Barhl2 protein sequences are arranged in correspondence with the evolution of the species, while the retinal expression of Barhl2 has been conserved throughout the animal kingdom. This could be related to changes in function or regulation of barhl1 paralogs that led to diversification and, in some cases, retention rather than loss of a duplicate. Unfortunately, without access to expression information for Tetraodon and stickleback, no further hypothesis on the relationship of function and copy number can be made.

In line with these observations, our in situ hybridization analysis suggests that the zebrafish barhl2 expression pattern closely resembles that of barhl2 ortholog in other vertebrates, and therefore might play a role in the same neuronal lineages. The mouse Barhl2 takes part in the specification of the ACs, and later aspects of RGC maturation $[9,10]$. Functional experiments will be necessary to test this hypothesis in zebrafish. It also remains to be demonstrated whether the expression of barhl2 within the GCL is restricted to displaced ACs or RGCs or both, and whether part of barhl2 expression might be dependent on atoh7, like in other vertebrates [9]. As the expression of atoh7 and barhl 2 are mostly complementary, our hypothesis is that barhl2 transcriptional 


\section{A}

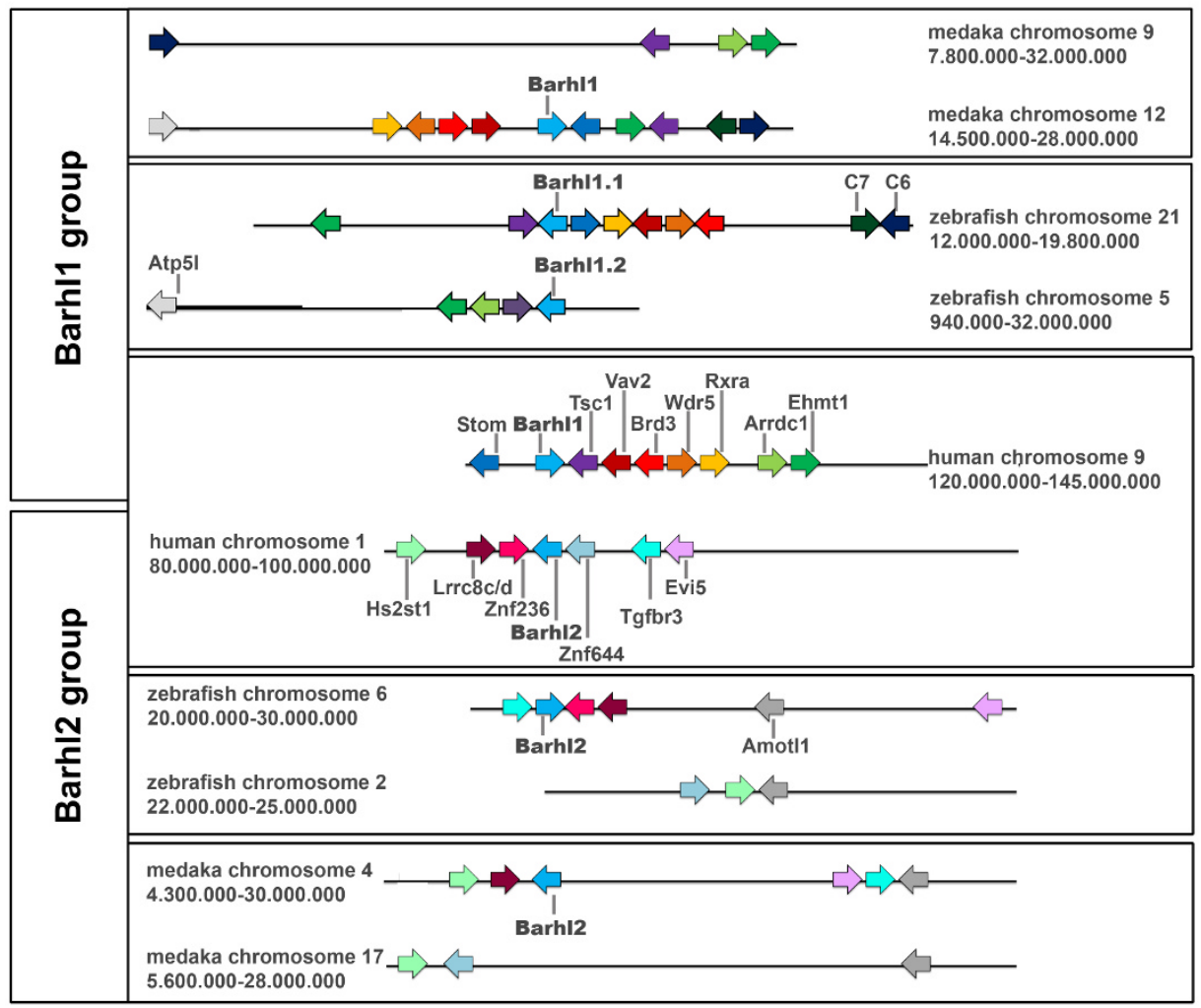

B

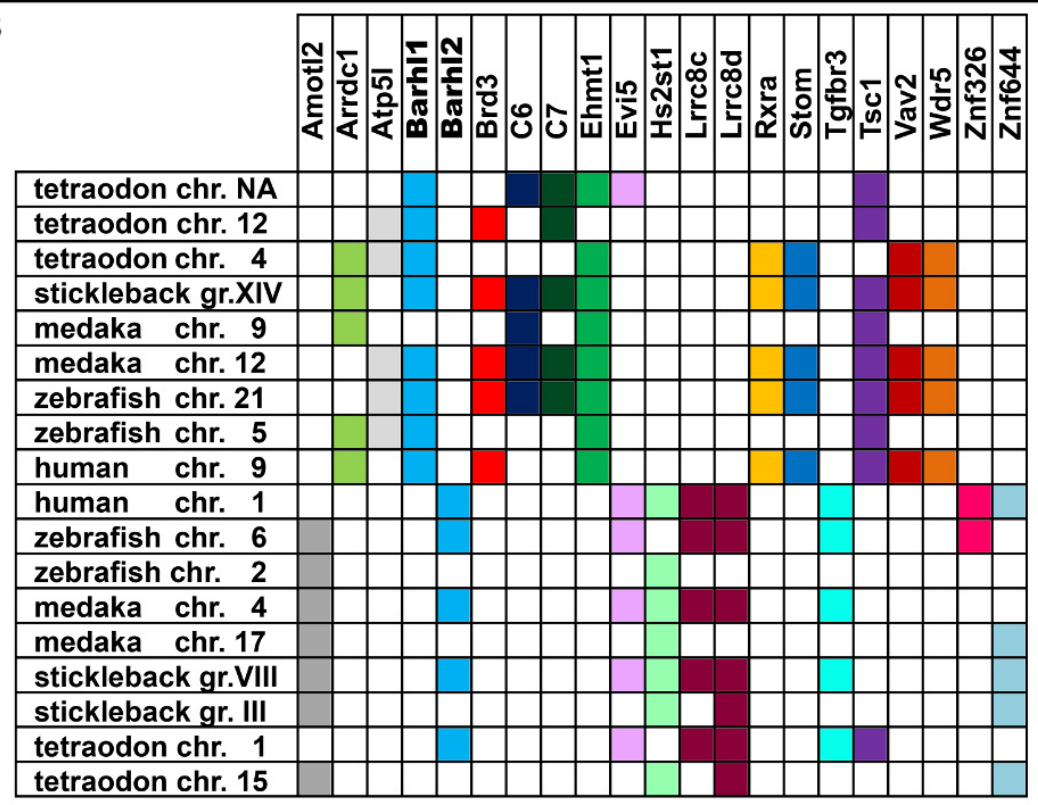

Figure 9 Synteny conservation in the barhl locus. (A) Chromosomal regions carrying barhl in human, zebrafish and medaka were compared and neighbouring genes with conserved synteny identified. Each gene is represented in a different colour as an arrow showing its orientation on the chromosome. Neighbouring genes that are in synteny are shown with a schematic indication of their distance; which is not in scale. Exact location and full name of each gene can be found in the Additional file 1:Table 1. (B) Comparison of gene distribution in the ten loci for four teleost species and human. Syntenic genes are found either in the barhl1 group or the barhl2 group. 
activation in the AC lineage is independent from Atoh7. Other candidate factors might be at work in inducing barhl2, particularly those factors that have been shown to favour AC fate at the expense of RGCs in zebrafish [15]. The striking complementary expression of atoh7 and barhl2 that we observed (see Figure 4) would rather supports a negative feedback between the two genes. Interestingly, similar mutually excluding expression domains have been found also in Drosophila between the homolog atonal and bar genes [32]. In this study, a negative feedback has been shown to occur between the two Drosophila genes, thus suggesting the conservation of an ancestral feature. The expression of barhl2 in the zebrafish atoh7-/- retina at $40 \mathrm{hpf}$ appears to be retained in time but its pattern of expression is shifted towards the lens (Figure 5B). Most ACs born at this stage become displaced in the presumptive GCL (which is devoid of RGCs, [19]); which might account for the localization of barhl2 expression in this domain. Thus, our current data doesn't provide, neither it excludes evidences for negative feedback between atoh7 and barhl2 in zebrafish. In contrast to barhl1.1, we found that barhl1.2 is expressed in the zebrafish retina. Surprisingly, this expression occurs nearly in synchrony with the one of atoh7 and the development of RGCs. Similarly, we found that the medaka barhl1 spatio-temporal expression is reminiscent of what has been previously described for atoh7 expression in the medaka retina, being first detected at stage 25 and becoming restricted to the CMZ at stage 30-35 [26]. This observation further supports the hypothesis of conserved dynamics of retinal lineages specification in both fish "twins", at least with respect to atoh 7 and barhl genes. Thus, due to this extreme similarity between barhl1.2 and atoh7 expression within the RGC lineage, it is also possible that Barhl1.2 function became redundant with respect to the one of Atoh7 and was therefore lost in the tetrapod lineage. In light of this intriguing hypothesis, it will be very interesting to ask what is the retained function of the zebrafish barhl1.2 in the RGC lineage and in relation to atoh7.

After branching of the metazoan clades into protostomes and deuterostomes, a tandem replication in Drosophila melanogaster led to the existence of two Bar factors in the fruit fly that are assumed to be redundant. In other insects, such as Drosophila ananassae, Anopheles or Apis melifera, only one Bar can be found (results of TBLASTN search). In the line of the deuterostomes, at least one round of WGD took place before the rise of the gnathostomes (jawed vertebrates). We assume that before this period of intense genome rearrangement, there was only one Barhl, as we can find in the genome sequences of Branchiostoma floridae (amphioxus) or Ciona savignyi (sea squirt, results of
TBLASTN search). Going further up in the evolutionary tree, at least two Barhls could be found in every species we looked at. These observations raise the intriguing hypothesis that Barhl factors have evolved in complexity proportional to the evolving diversity of retinal arrangement and rhabdomeric photoreceptor-derived cell types [33]. In summary, our study provides an example on how retained gene paralogs might have evolved in contributing to the specification of distinct cell-lineages in the vertebrate retina. A larger scale analysis of the functional implications of the differences in retinal key transcription factors between teleosts and other vertebrates will help to speculate further on the role of duplication retention in the evolution of vertebrates, and more specifically during eye evolution.

\section{Conclusions}

In summary, our teleost comparison of Barhl orthologs highlights differences in expression patterns within retinal cell populations and regarding Atoh7 regulation. By extensive cross-species analysis of the barhl loci, these differences could be linked to differential selective pressure: while the barhl2 locus remains under evolutionary constraint, we show that the barhl1 locus rapidly evolves, thereby leading to functional differences within barhl1 paralogs. Our cross-species analysis provides insights on how retained gene paralogs might have evolved in relation to distinct cell-lineages in the vertebrate retina.

\section{Methods}

\section{Fish lines}

Breeding and rising of zebrafish followed standard protocols [34]. Zebrafish embryos were treated with 0.0045\% 1-Phenyl-2-Thiourea (PTU) in medium after gastrulation to prevent pigment formation. Medaka embryos were kept in ERM medium containing $1 \mathrm{~g} / \mathrm{l}$ $\mathrm{NaCl}, 30 \mathrm{mg} / \mathrm{l} \mathrm{KCl}, 40 \mathrm{mg} / \mathrm{l} \mathrm{CaCl} 2 \times 2 \mathrm{H} 2 \mathrm{O}$ and 163 $\mathrm{mg} / \mathrm{l} \mathrm{MgSO} 4 \times 7 \mathrm{H} 2 \mathrm{O}$ in deionized water.

\section{Ethics statement}

All fish are housed in the fish facility of our laboratory, which was built according to the local animal welfare standards (Tierschutzgesetz 111, Abs. 1, Nr. 1) and in accordance with European Union animal welfare guidelines. The facility is under the supervision of the local representative of the animal welfare agency. No animal experiments were performed. Embryos of medaka (Oryzias latipes) and zebrafish (Danio rerio) were used exclusively at stages prior to hatching (not considered as animals according to German law and European union regulations). Zebrafish and medaka were raised and maintained as described previously [26]. The following strains were used for zebrafish embryos: wild type WIK/ $\mathrm{AB}$ and for medaka embryos: the Cab wild type strain." 


\section{Amplification and cloning of medaka Barhl2}

An 800 bp fragment was polymerase chain reaction (PCR)-amplified from mixed stage medaka cDNA eye and brain using the forward primer (GAGATAGACACCGTGGGAACTGG) and reverse primer (CTGATGGAGTCCGGTACATGCTG) designed to bind in exons 1 and 4 of olbarhl2 (ENSORLT00000002844, EnsEMBL v58). Cycling conditions: five cycles $95^{\circ} \mathrm{C}, 10 \mathrm{sec}, 65^{\circ} \mathrm{C}, 20 \mathrm{sec}, 72^{\circ} \mathrm{C}, 4$ min; followed by 28 cycles with annealing at $60^{\circ} \mathrm{C}$. A Taq DNA-Polymerase was used for A-Tailing, incubation was for $30 \mathrm{~min}$ at $72^{\circ} \mathrm{C}$. The PCR product was cloned into pCRII TOPO TA vector (Invitrogen) and sequenced using T7 and SP6 promoters. The sequence has been submitted to the EMBL [GenBank: JQ008931].

\section{Whole-mount in situ hybridization}

Single whole-mount in situ hybridization of barhl genes was carried out as previously described in [30], for the zebrafish embryos and in [31], for the medaka embryos. Riboprobes where labelled with digoxigenin-UTP (Roche Applied Science). Hybridization with the probe was carried on over night at $65^{\circ} \mathrm{C} / 68^{\circ} \mathrm{C}$. Anti-DIG primary antibody coupled to alkaline phosphatase (Roche Molecular Biochemicals) and NBT-BCIP (Roche Molecular Biochemicals) was used for signal detection. For the FISH on zebrafish embryos, modifications were applied to the method described in [30], as suggested by Stephan Kirchmaier (unpublished). Embryos were washed with $100 \mu \mathrm{l}$ Tyramide Signal Amplification (TSA) solution and incubated with FITC in TSA. Incubation with the barhl 2 probe was for 30 minutes, incubation with the atoh7 probe was for 40 minutes. Embryos were then kept in the dark for all following steps. For detection and staining of the antisense probes, embryos were washed $5 \times 10 \mathrm{~min}$ with TNT $(0.1 \mathrm{M}$ Tris $\mathrm{pH} 7.5,0.15 \mathrm{M}$ $\mathrm{NaCl}, 0.1 \%$ Tween 20), incubated with $1 \% \mathrm{H} 2 \mathrm{O} 2$ in TNT for $20 \mathrm{~min}$ and washed again $5 \times 10 \mathrm{~min}$. Embryos were blocked in TNB (2\% DIG Block in TNT) for $1 \mathrm{~h}$ at RT and afterwards incubated with Anti-Digoxigenin-POD Fab fragments diluted 1:100 in TNB. For signal detection, Fluorescein (FITC), Cyanine 3 (Cy3) or Cyanine 5 (Cy5) Fluorophore Tyramide by PerkinElmer was used. Embryos were then incubated in $1 \times 4,6$-Diamidin-2phenylindol (DAPI) in TNT over night at $4^{\circ} \mathrm{C}$ and washed several times in TNT the next day. Embryos stained with NBT/BCIP were mounted in $87 \%$ Glycerol on microscope slides and imaged with a Leica DM5000B, 10x or 20x air objectives, Leica CD500 microscope camera and Leica FireCam 1.7.1 software. Double fluorescent embryos were mounted in $100 \times 15$ $\mathrm{mm}$ glass bottom dishes in $1.5 \%$ low melting agarose. Confocal stacks were taken using the Leica SP5 confocal microscope, 20x water immersion objective and Leica Application Suite (LAS) software. FITC was excited at
$488 \mathrm{~nm}$ by Argon laser, Cy3 by the $568 \mathrm{~nm}$ HeliumNeon laser, Cy5 at $633 \mathrm{~nm}$ by Helium-Neon and DAPI by an UV laser. Emission was sensed at 500-550 nm for FITC, 650-700 nm for Cy3, 650-800 nm for Cy5 and 400-500 nm for DAPI. Emission channels were imaged sequentially to avoid bleed-through of the two fluorescent signals. Pictures were processed using the open source software ImageJ version 1.43 and Adobe Photoshop CS3.

\section{Phylogenetic analysis}

Protein sequences were obtained from EnsEMBL Genome Browser (v58) after using TBLASTN to perform a search for the zebrafish protein sequence of Barhl2 and Barhl1.1, respectively, against the genomic DNA of the used species to check the integrity of the annotated protein sequences. The following protein sequences were used: Danio rerio Barhl1.1 (Accession number ENSDARP00000016114) Danio rerio Barhl1.2 (Acc. No. ENSDARP00000051473) Danio rerio Barhl2 (Acc. No. ENSDARP00000093436) Homo sapiens BARHL1 (Acc. No. ENSP00000263610) Homo sapiens BARHL2 (Acc. No. ENSP00000359474) Mus musculus BARHL1 (Acc. No. ENSMUSP00000053147) Mus musculus BARHL2 (Acc. No. ENSMUSP00000084005) Xenopus tropicalis barhl1 (Acc. No. ENSXETP00000013720) Xenopus tropicalis barhl2 (Acc. No. ENSXETP00000051744) Gasterosteus aculeatus Barhl1 (ENSGACP00000023974) Gasterosteus aculeatus Barhl2 (ENSGACP00000005730) Tetraodon nigroviridis Barhl2 (ENSTNIP00000016761) Tetraodon nigroviridis Barhl1.1 (ENSTNIP00000008103) Tetraodon nigroviridis Barhl1.2 (ENSTNIP00000008179) Tetraodon nigroviridis Barhl1.3 (ENSTNIP00000008783) Takifugu rubripes Barhl1 (ENSTRUP00000013477) Takifugu rubripes Barhl2 (ENSTRUP00000032575) Oryzias latipes Barhl1 (Acc. No. ENSORLP00000015485) Ciona savignyi Barhl (ENSCSAVP00000019219) Drosophila melanogaster Bar1 (Acc. No. FBpp0074204) Drosophila melanogaster Bar2 (Acc. No. FBpp00742043) Sequences obtained from National Center for Biotechnology's (NCBI) GenBank database (Release 178): Xenopus laevis Barhl1 (Acc. No. AAG14451.1) Xenopus laevis Barhl2 (Acc. No. NP_001082021.1) Salmo salar Barhl1 (Acc. No. NP_001167081.1) Branchiostoma floridae Barhl1 (XP_002596391.1). The protein sequence of Oryzias latipes Barhl2 was obtained from translating the sequenced cDNA clone using the ExPASy online translate tool from Swiss Institute of Bioinformatics (SIB). Alignment of the sequences was produced using MUSCLE online at the European Bioinformatics Institute (EBI) [35]. A phylogenetic tree was assembled using BioNJ online at phylogeny.fr performing 1000 bootstraps and using Jones-Taylor-Thornton matrix. This distancebased algorithm is claimed to be well suited for 
comparison of sequences with high substitution rates $[36,37]$. The tree was visualized using the open source software Dendroscope [38].

\section{Synteny analysis}

Chromosomal loci of barhl genes in human, zebrafish, stickleback, Tetraodon and medaka were compared by identifying all genes that occur in more than one of the loci. The position of each of these genes was then searched in all species using the EnsEMBL database search function (EnsEMBL release v58). Position and identity of genes were plotted schematically according to their order and orientation (Figure 9, not to scale). Exact position of each gene can be found in the Additional file 1: Table 1.

\section{Additional material}

Additional file 1: Table 1: Genomic location of genes used for the syntenic analysis. Genomic locations according to EnsEMBL Database release 60 . The numbers indicate chromosome number:location on chromosome:strand ( 1 = sense, $-1=$ antisense). Un_random indicates sequences that have not been allocated to a specific chromosome.

\section{Acknowledgements}

We would like to thank S. Kirchmaier for providing the double FISH protocol; M. Eichenlaub for providing the medaka CDNA and helping with the in situ hybridization; B. Wittbrodt, E. Leist, A. Saraceno, M. Majewski, B. Seiferling, T. Kellner, L. Schertel, C. Mueller for fish maintenance and technical assistance. We are grateful to L. Ettwiller, M. Eichenlaub, P. Jusuf, G. Lupo, M. Carl, W.A. Harris, S. Schneider and J. Wittbrodt for discussion and suggestions on the manuscript, and to J. Wittbrodt for generous support. SA is funded by LGFG (Funding program of the State of Baden-Württemberg). This work was supported by the DFG (Eigenen Stelle to LP).

\section{Author details}

${ }^{1}$ Centre for Organismal Studies, University of Heidelberg, Heidelberg 69120, Germany. ${ }^{2}$ The Victor Chang Cardiac Research Institute, Darlinghurst NSW 2010, Australia.

\section{Authors' contributions}

LP conceived and supervised the study. LNS, LP and SA designed the experiments. LNS and SA performed and analyzed all the experiments presented. LP and LNS wrote the manuscript. MR contributed with the phylogenetic and synteny analysis, data interpretation, and to writing the manuscript. All authors revised and approved the final manuscript.

Received: 12 June 2011 Accepted: 21 November 2011

Published: 21 November 2011

\section{References}

1. Wang JC, Harris WA: The role of combinational coding by homeodomain and bHLH transcription factors in retinal cell fate specification. Dev Biol 2005, 285:101-115

2. Goode DL, Cooper GM, Schmutz J, Dickson M, Gonzales E, Tsai M, Karra K, Davydov E, Batzoglou S, Myers RM, Sidow A: Evolutionary constraint facilitates interpretation of genetic variation in resequenced human genomes. Genome Res 2010, 20:301-310.

3. Force A, Lynch M, Pickett FB, Amores A, Yan YL, Postlethwait J: Preservation of duplicate genes by complementary, degenerative mutations. Genetics 1999, 151:1531-1545.

4. Prince VE, Pickett FB: Splitting pairs: the diverging fates of duplicated genes. Nat Rev Genet 2002, 3:827-837.
5. Kawauchi D, Muroyama Y, Sato T, Saito T: Expression of major guidance receptors is differentially regulated in spinal commissural neurons transfated by mammalian Barh genes. Dev Biol 2010, 344:1026-1034.

6. Reig G, Cabrejos ME, Concha ML: Functions of BarH transcription factors during embryonic development. Dev Biol 2007, 302:367-375.

7. Patterson KD, Cleaver O, Gerber W, White FG, Krieg PA: Distinct expression patterns for two Xenopus Bar homeobox genes. Dev Genes Evol 2000, 210:140-144

8. Saito T, Sawamoto K, Okano H, Anderson DJ, Mikoshiba K: Mammalian BarH homolog is a potential regulator of neural bHLH genes. Dev Biol 1998, 199:216-225.

9. Ding $Q$, Chen $H$, Xie X, Libby RT, Tian N, Gan L: BARHL2 differentially regulates the development of retinal amacrine and ganglion neurons. J Neurosci 2009, 29:3992-4003.

10. Mo Z, Li S, Yang X, Xiang M: Role of the Barhl2 homeobox gene in the specification of glycinergic amacrine cells. Development 2004, 131:1607-1618

11. Poggi L, Vottari T, Barsacchi G, Wittbrodt J, Vignali R: The homeobox gene Xbh1 cooperates with proneural genes to specify ganglion cell fate within the Xenopus neural retina. Development 2004, 131:2305-2315.

12. Colombo A, Reig G, Mione M, Concha ML: Zebrafish BarH-like genes define discrete neural domains in the early embryo. Gene Expr Patterns 2006, 6:347-352.

13. Furlong RF, Holland PW: Were vertebrates octoploid? Philos Trans R SoC Lond B Biol Sci 2002, 357:531-544

14. Poggi L, Carl M, Vignali R, Barsacchi G, Wittbrodt J: Expression of a medaka (Oryzias latipes) Bar homolog in the differentiating central nervous system and retina. Mech Dev 2002, 114:193-196.

15. Jusuf PR, Almeida AD, Randlett O, Joubin K, Poggi L, Harris WA: Origin and determination of inhibitory cell lineages in the vertebrate retina. J Neurosci 2011, 31:2549-2562.

16. Masai I, Stemple DL, Okamoto H, Wilson SW: Midline signals regulate retinal neurogenesis in zebrafish. Neuron 2000, 27:251-263.

17. Brown NL, Patel S, Brzezinski J, Glaser T: Math5 is required for retinal ganglion cell and optic nerve formation. Development 2001, 128:24972508.

18. Ghiasvand NM, Rudolph DD, Mashayekhi M, Brzezinski JAt, Goldman D, Glaser T: Deletion of a remote enhancer near ATOH7 disrupts retinal neurogenesis, causing NCRNA disease. Nat Neurosci 2011, 14:578-586.

19. Kay JN, Finger-Baier KC, Roeser T, Staub W, Baier H: Retinal ganglion cell genesis requires lakritz, a Zebrafish atonal Homolog. Neuron 2001, 30:725-736.

20. Wang SW, Kim BS, Ding K, Wang H, Sun D, Johnson RL, Klein WH, Gan L: Requirement for math 5 in the development of retinal ganglion cells. Genes Dev 2001, 15:24-29.

21. Feng L, Xie ZH, Ding Q, Xie X, Libby RT, Gan L: MATH5 controls the acquisition of multiple retinal cell fates. Mol Brain 2010, 3:36.

22. Poggi L, Vitorino M, Masai I, Harris WA: Influences on neural lineage and mode of division in the zebrafish retina in vivo. J Cell Biol 2005, 171:991-999.

23. Furutani-Seiki M, Wittbrodt J: Medaka and Zebrafish, an evolutionary twin study. Mech Dev 2004, , 7-8: 629-637.

24. Peden E, Kimberly E, Gengyo-Ando K, Mitani S, Xue D: Control of sexspecific apoptosis in $\mathrm{C}$. elegans by the BarH homeodomain protein $\mathrm{CEH}$ 30 and the transcriptional repressor UNC-37/Groucho. Genes Dev 2007, 21:3195-3207.

25. Iwamatsu T: Stages of normal development in the medaka Oryzias latipes. Mech Dev 2004, 7-8: 605-618

26. Souren M, Martinez-Morales JR, Makri P, Wittbrodt B, Wittbrodt J: A global survey identifies novel upstream components of the Ath5 neurogenic network. Genome Biology 2009, 10(9):R92

27. Kikuta H, Laplante M, Navratilova P, Komisarczuk AZ, Engstrom PG, Fredman D, Akalin A, Caccamo M, Sealy I, Howe K, et al: Genomic regulatory blocks encompass multiple neighboring genes and maintain conserved synteny in vertebrates. Genome Res 2007, 17:545-555.

28. Ravi V, Venkatesh B: Rapidly evolving fish genomes and teleost diversity. Curr Opin Genet Dev 2008, 18:544-550.

29. Kuraku S, Meyer A: The evolution and maintenance of Hox gene clusters in vertebrates and the teleost-specific genome duplication. Int J Dev Biol 2009, 53:765-773.

30. Panopoulou G, Poustka AJ: Timing and mechanism of ancient vertebrate genome duplications - the adventure of a hypothesis. Trends Genet 2005, 21:559-567. 
31. Lim J, Choi KW: Induction and autoregulation of the anti-proneural gene Bar during retinal neurogenesis in Drosophila. Development 2004, 131:5573-5580.

32. Lim J, Choi KW: Bar homeodomain proteins are anti-proneural in the Drosophila eye: transcriptional repression of atonal by Bar prevents ectopic retinal neurogenesis. Development 2003, 130:5965-5974.

33. Arendt D: Evolution of eyes and photoreceptor cell types. Int J Dev Biol 2003, 47:563-571.

34. Westerfield M: The zebrafish book 1994.

35. Edgar RC: MUSCLE: multiple sequence alignment with high accuracy and high throughput. Nucleic Acids Res 2004, 32:1792-1797.

36. Dereeper A, Guignon V, Blanc G, Audic S, Buffet S, Chevenet F, Dufayard JF, Guindon S, Lefort V, Lescot M, et al: Phylogeny.fr: robust phylogenetic analysis for the non-specialist. Nucleic Acids Res 2008, 36:W465-469.

37. Gascuel O: BIONJ: an improved version of the $\mathrm{NJ}$ algorithm based on a simple model of sequence data. Mol Biol Evol 1997, 14:685-695.

38. Huson DH, Richter DC, Rausch C, Dezulian T, Franz M, Rupp R:

Dendroscope: An interactive viewer for large phylogenetic trees. $B M C$ Bioinformatics 2007, 8:460.

doi:10.1186/1471-2148-11-340

Cite this article as: Schuhmacher et al: Evolutionary relationships and diversification of barhl genes within retinal cell lineages. BMC

Evolutionary Biology 2011 11:340.

\section{Submit your next manuscript to BioMed Central} and take full advantage of:

- Convenient online submission

- Thorough peer review

- No space constraints or color figure charges

- Immediate publication on acceptance

- Inclusion in PubMed, CAS, Scopus and Google Scholar

- Research which is freely available for redistribution

Submit your manuscript at www.biomedcentral.com/submit 